\title{
COVID-19-Related Weight Gain in School-Aged Children
}

\author{
Mohammadreza Rezaeipour (iD) 1, \\ ${ }^{1}$ Department of Sports Science, Faculty of Educational Sciences and Psychology, University of Sistan and Baluchestan, Zahedan, Iran \\ "Corresponding author: Department of Sports Science, Faculty of Educational Sciences and Psychology, University of Sistan and Baluchestan, P. O. Box: 9816745639, Zahedan, \\ Iran. Tel: +98-9153414047. Email: rezaeipour@ped.usb.ac.ir \\ Received 2020 October 26; Revised 2020 December 15; Accepted 2020 December 15.
}

Keywords: Coronavirus Disease 2019, COVID-19, Nutrition, Pandemic, Public Health, SARS-CoV, Weight Gain

\section{Dear Editor,}

This letter describes a proposed method to prevent children from gaining weight during not attending school due to COVID-19. The pandemic of Coronavirus Disease 2019(COVID-19) is making considerable mortality and morbidity, putting pressure on health care systems, winding down the economy, and increasing school absenteeism. While lowering its immediate impact is a priority, I want to focus on a long-term impact on the health of children. By not attending school and staying at home, COVID-19 may lead to a stepped-up children's obesity epidemic and increases obesity disorders. The COVID-19 pandemic has prevented students from attending school, forcing them to stay at home in many parts of Iran. The school system is not expected to return to normal this school year. Experiences gained in the Middle East countries such as Iran indicate that the observance of social distancing if canceled after a short time, must be periodically re-established its orders to control the prevalence of COVID-19 $(1,2)$. In summary, the COVID-19 pandemic in Iran is expected to intensify dramatically this year and increase school absenteeism, which will make worse the risk factors for weight gain related to summer vacations.

Although a lot of literature is available about poor eating habits and body exercises in schools, according to research, children experience an increase in unhealthy weight not within the school year but generally in the summer months when they do not attend school (3-5). A study during school years has shown that the outbreak of overweight/obesity in students occurred only within the summer vacations (3). Research and follow-up studies show that an abnormal increase in weight is evident at school summer vacation, especially for some breeds, as well as children who were already extra weight $(3,5)$. It is worth noting that the data obtained indicate that the weight increased during the summer months remains throughout the school year and accumulates from summer to summer (5). Abnormal weight gain in childhood is a long-standing problem, as another study showed that childhood obesity is related to adulthood weight gain (6). For example, an abnormal increase in weight at the age of five was significantly linked to an increase in body mass index and fat mass in middle age (6).

I argue that with raising out-of-school time, the pandemic of COVID-19 aggravates all risk factors for abnormal weight gain due to summer vacations (7). Not attending school, staying at home, and on-place shelter orders challenge children in the field of nutrition and physical exercise. On the one hand, physical activity and doing it in groups are limited for children, and on the other hand, they are provided with a variety of food resources in terms of entertainment. Predictions consistent with the culture and home facilities of Iranians demonstrate that not attending school can lead to more eating foods (8), junk foods, and/or miss meals (8) among children of school age. Food insecurity is associated with the risk of abnormal increases in weight, and I also anticipate that children's food insecurity will increase in this epidemic $(9,10)$.

Additionally, while families keep stable foods on the shelf, they seem to buy junk food, processed products, and high-calorie comfort diets (11). Supermarket shopping experience demonstrates that along with shelves containing flour, beans, and rice, shelves containing ramen noodles, chips, crackers, sugary flakes, soft drinks, and ready-to-eat processed products are empty. Although supplying shelfstable food is an obvious need for preparation and helps minimize out-of-home trips, as mentioned above, I predict that lots of children will be faced with higher-calorie diets while replying to the pandemic.

The orders of social spacing and staying at home reduce the opportunity for children's exercise in the world, especially for urban children where they are in the lim- 
ited space of apartments. The inactive lifestyle and watching time are anticipated to develop according to the social distance guidelines. Research demonstrates that the use of online games is now growing rapidly (12). Watching time in childhood is associated with overweight/obesity, possibly due to dual sedentary problems and the connection between watching time and snacking $(13,14)$. Whereas inactive lifestyles involve all children, it is likely to have the greatest influence on urban children who lack access to places where they can sustain social distancing. Playgrounds and parks are open up in some cities, but the public believes that keeping them clean is impossible, and it is difficult to maintain social distancing for children. It is, therefore, clear that urbanized households may not utilize these sites, and this exacerbates the discrepancy among those who may/may not be physically active outside the home.

What are the health programs and public health services that can be recommended during this pandemic to protect healthy eating and active school-aged lifestyles? First, it needs innovative ways to deal with food insecurity in restrictions of social distancing or complete residence at home. To reduce fast food consumption and its harms to all family members, it is proposed to have a special look at the Centre Farmers' Market that produce local and home-cooked healthy foods along with respecting the health protocols (15). Alternatively, by increasing the capacity of distance learning in schools, we must give priority to physical education. In places where schools send home some curricula, they can also send home physical education curricula. There are various exercise plans intended for business travelers in hotel rooms with finite sports equipment that can be adjusted to the home curriculum (16). For schools where online classes are possible, sports teachers can broadcast physical education classes. Besides, there are currently over 300,000 health-related applications around the world (17). Some of these apps supply homemade exercise plan with training (like videos, photos, and explanatory text) and offers about the number of sets and reps, rest time between various exercises, etc. Technology-based exercises such as active video games, which need bodily mobility to perform them, can also be used (18). These active video games supply some activities in the field of muscle-building, fitness, dance, martial arts, and various sports.

Lastly, studies can be conducted to investigate obesity in Iranian children due to absenteeism in the school during the COVID-19 epidemic, and their results can be used to prevent undesirable

\section{Footnotes}

Authors' Contribution: Rezaeipour M. was involved in the study concept and design, drafting of the manuscript, critical revision of the manuscript, and finalizing the manuscript for important intellectual content.

Conflict of Interests: No conflicts of interest are declared.

Funding/Support: No fund or support was received for the production of this manuscript.

\section{References}

1. Ghanbari B. On forecasting the spread of the COVID-19 in Iran: The second wave. Chaos Solitons Fractals. 2020;140:110176. doi: 10.1016/j.chaos.2020.110176. [PubMed: 32834656]. [PubMed Central: PMC7386426].

2. Ayyoubzadeh SM, Ayyoubzadeh SM, Zahedi H, Ahmadi M, R. Niakan Kalhori S. Predicting COVID-19 Incidence Through Analysis of Google Trends Data in Iran: Data Mining and Deep Learning Pilot Study. JMIR Public Health Surveill. 2020;6(2). e18828. doi: 10.2196/18828. [PubMed: 32234709]. [PubMed Central: PMC7159058].

3. Tanskey LA, Goldberg J, Chui K, Must A, Sacheck J. The State of the Summer: a Review of Child Summer Weight Gain and Efforts to Prevent It. Curr Obes Rep. 2018;7(2):112-21. doi: 10.1007/s13679-018-0305-z. [PubMed: 29644576]. [PubMed Central: PMC6857933].

4. Weaver RG, Beets MW, Brazendale K, Brusseau TA. Summer Weight Gain and Fitness Loss: Causes and Potential Solutions. Am J Lifestyle Med. 2019;13(2):116-28. doi: 10.1177/1559827617750576. [PubMed: 30800015]. [PubMed Central: PMC6378497].

5. Yuca SA. Childhood obesity. BoD-Books on Demand; 2012.

6. Rundle AG, Factor-Litvak P, Suglia SF, Susser ES, Kezios KL, Lovasi GS, et al. Tracking of Obesity in Childhood into Adulthood: Effects on Body Mass Index and Fat Mass Index at Age 50. Child Obes. 2020;16(3):22633. doi: 10.1089/chi.2019.0185. [PubMed: 32191541]. [PubMed Central: PMC7099417].

7. Rundle AG, Park Y, Herbstman JB, Kinsey EW, Wang YC. COVID19-Related School Closings and Risk of Weight Gain Among Children. Obesity (Silver Spring). 2020;28(6):1008-9. doi:10.1002/oby.22813. [PubMed: 32227671]. [PubMed Central: PMC7440663].

8. Azizi-Soleiman F, LMotlagh ME, Qorbani M, Heshmat R, Ardalan G, Mansourian M, et al. Dietary habits and health related behaviors in Iranian children and adolescents: the CASPIAN-IV study. Int J Pediatr. 2016;4(7):2087-97.

9. Brown AGM, Esposito LE, Fisher RA, Nicastro HL, Tabor DC, Walker JR. Food insecurity and obesity: research gaps, opportunities, and challenges. Transl Behav Med. 2019;9(5):980-7. doi:10.1093/tbm/ibz117. [PubMed: 31570918]. [PubMed Central: PMC6937550].

10. Oberle MM, Romero Willson S, Gross AC, Kelly AS, Fox CK. Relationships among Child Eating Behaviors and Household Food Insecurity in Youth with Obesity. Child Obes. 2019;15(5):298-305. doi: 10.1089/chi.2018.0333. [PubMed: 31090441].

11. Skerritt J, Mulvay L, Almeida I. Americans drop kale and quinoa to lock down with chips and oreos. Bloomberg News; 2020, [cited March 21, 2020]. Available from: https:/www.bloomberg.com/news/articles/ 2020-03-21/americans-drop-kale-and-quinoa-to-lock-downwith-chips-and-oreos.

12. Wilde T. Online gaming surge: steam breaks concurrent user record amid social distancing mandates. GeekWire website; 2020, [cited March 16, 2020]. Available from: https://www.geekwire.com/2020/onlinegaming-surge-steam-breaks-concurrent-user-record-amid-socialdistancing-mandates/. 
13. Ghobadi S, Totosy de Zepetnek JO, Hemmatdar Z, Bellissimo N, Barat $\mathrm{R}$, Ahmadnia $\mathrm{H}$, et al. Association between overweight/obesity and eating habits while watching television among primary-school children in the city of Shiraz, Iran. Public Health Nutr. 2018;21(3):571-9. doi: 10.1017/S1368980017003251. [PubMed: 29173231].

14. Mazur A, Caroli M, Radziewicz-Winnicki I, Nowicka P, Weghuber D, Neubauer D, et al. Reviewing and addressing the link between mass media and the increase in obesity among European children: The European Academy of Paediatrics (EAP) and The European Childhood Obesity Group (ECOG) consensus statement. Acta Paediatr. 2018;107(4):568-76. doi:10.1111/apa.14136. [PubMed: 29164673].

15. Ruelas V, Iverson E, Kiekel P, Peters A. The role of farmers' mar- kets in two low income, urban communities. J Community Health. 2012;37(3):554-62. doi: 10.1007/s10900-011-9479-y. [PubMed: 21922162].

16. Team G. 10 Bodyweight Exercises You Can Do Anywhere. Power. 2019;30.

17. Gordon WJ, Landman A, Zhang H, Bates DW. Beyond validation: getting health apps into clinical practice. NPJ Digit Med. 2020;3:14. doi: 10.1038/s41746-019-0212-z. [PubMed: 32047860]. [PubMed Central: PMC6997363].

18. Street TD, Lacey SJ, Langdon RR. Gaming Your Way to Health: A Systematic Review of Exergaming Programs to Increase Health and Exercise Behaviors in Adults. Games Health J. 2017;6(3):136-46. doi: 10.1089/g4h.2016.0102. [PubMed: 28448175]. 